\title{
La investigación en educación física. Una postura epistemológica desde la complementariedad etnográfica
}

Physical education research: an epistemological view from etnographical complementarity

\author{
Napoleón Murcia Peña
}

\section{Resumen}

El artículo aborda el problema de la investigación en la educación física, la cual ha estado fuertemente influenciada por los imaginarios instrumentales del racionalismo cartesiano y el positivismo comptiano: Estos imaginarios epistemológicos y metodológicos han dejado una huella profunda en la consolidación de la dependencia social y la heteronomía individual, y que hoy se reconocen como insuficientes para abordar la compleja trama del mundo social y humano. Además, hace referencia a la realidad compleja de la educación física, en la cual se funden, como magmas efervescentes, lo sicosomático, lo social y lo histórico. Por tanto, es improcedente abordar el área desde imaginarios epistémicos y metodológicos unidimensionales y simples.

En el sentido anteriormente argumentado, se propone la complementariedad etnográfica como una alternativa para abordar esa realidad; de no ser así, se corre el riesgo de quedarse viendo al ser humano como un ser simple que puede fraccionarse indistintamente sin considerar siquiera su naturaleza autoeco organizada.

Investigación, educación física, postura epistemológica, complementariedad etnográfica.

\section{Abstract}

The article approaches the problem of research in Physical education, which has been strongly influenced by the instrumental imaginaries of Cartesian rationalism and Comptian positivism. These epistemological and methodological imaginaries have deeply influenced the building of social dependence and individual heteronomy. Nowadays, they are considered insufficient to approach the complex fabric of the social human world. The article also refers to the complex reality of physical education, where the psychosomatic, social and historical aspects are melted, as effervescent magmas. As a consequence, it is stated how unsuitable it is to approach this field from simple, unidimensional, epistemic and methodological imaginaries.

Ethnographical complementarity is posited as an alternative to approach this reality. Otherwise, there is a risk of seeing the human being as a simple being, indistinctly dividable, without any consideration as to its auto-echo organized nature.

Key words: $\quad$ Research, physical education, epistemological attitude, ethnographic complementarity.

Fecha de entrega: 5 de octubre de 2007

Fecha de aceptación: 9 de noviembre de 2007

* Licenciado en Educación Física. Doctor en Ciencias Sociales, Niñez y Juventud. Profesor de la Universidad de Caldas. Investigador de la Universidad de Caldas. Autor de Investigación cualitativa. napo@epm.net.co 


\section{Bases instrumentales de unos imaginarios positivistas}

Son dos las tendencias del siglo XIV que influyeron en la lógica nomológica de la ciencia; por un lado la tendencia inductiva de Bacon (Londres, 1561-1626), para quien el conocimiento procede de la experiencia, de las percepciones sensibles. Desde su perspectiva, se debe estudiar la naturaleza de las cosas utilizando el método inductivo que implica agrupar hechos, experimentarlos y comprobarlos para llegar a conceptos generales.

Por otro lado Descartes (Francia, 1596-1650), quien abre la matematización del mundo y con ella el idealismo representacional; desde la representación planabidimensional se funda la realización de la racionalidad como único escenario del conocimiento. La duda metódica propuesta por él no es otra cosa que asumir el mundo desde coordenadas estables que se van descartando hasta llegar a lo que se considera como el eje definitivo que genera el efecto estudiado.

Estas dos tendencias, heredadas desde Aristóteles y Platón, han marcado el conocimiento hasta nuestros días.

A partir de los aportes de estos dos autores se dieron los debates que originaron el siglo de las luces, la sociedad ilustrada del siglo XVIII. Es fuerte la influencia de estas dos tendencias en el realismo pedagógico que precede a la sociedad ilustrada. Rakte, en el siglo XIV llevó a la educación las ideas de Bacon; Comenio, en el mismo siglo aplicó el idealismo de Descartes, fundamentado en el representacionismo didáctico (Luzuriaga, 1976).

La mayoría de las razones del idealismo hicieron cuna en los imaginarios de la naciente sociedad ilustrada de ese siglo. Sin embargo, las influencias del inductivismo de Bacon se radicalizarían hacia el positivismo inductivo mediante los desarrollos de Augusto Comte (Montpelier, 1798-1846), quien muestra su marcado carácter positivista en sus dos grandes obras: Curso de filosofía positiva (1830) y Discurso sobre el espíritu positivo (1844).

En esta perspectiva positivista, el valor de la ciencia social está en la verificabilidad experimental y la generación de leyes universales. Justamente, ahí se ubican las críticas de sus detractores en el siglo XVII, como Voltaire y Rousseau, quienes no conciben el análisis de lo social desde los marcos de la ciencia positiva, que a partir de la observación de los fenómenos descubre, explica y experimenta el comportamiento en términos de leyes universales (estados sociales).

La fuerte influencia del idealismo exige el método experimental y la lógica matemática, como único méto- do válido (método nomológico) para lograr el conocimiento científico.

Estas dos posturas extremas condujeron a la neutralidad valorativa y con ella al aislamiento del ser humano, hasta desplazarlo del conocimiento que producía, circunstancia agudizada por teorías como el racionalismo lógico del Círculo de Viena.

Los desarrollos posteriores en la línea del positivismo ubican a Karl Raimund Popper (Viena, 1902-1994) como el crítico del positivismo decimonónico, al proponer el racionalismo crítico y la teoría de la falsación como método para superar el verificacionismo.

Desde Popper se comienza a inclinar la balanza hacia la importancia de lo que el ser hưmano haya desarrollado como antecedente, pues las hipótesis no son inductivas, sino que se construyen desde la deducción de teorías ya formuladas.

Pese a ello, Popper continúa buscando leyes universales y se ampara en el método nomológico; por tanto, es considerado por muchos como positivista, pese a sus críticas profundas.

En esta línea positivista, la orientación hacia el reconocimiento de lo humano en el conocimiento científico continúa con las críticas de Lakatos (Hungría, 1922-1974) a lo que llama "el falsacionismo ingenuo" de Popper y su propuesta de los programas de investigación científica en los cuales juega un papel de gran importancia la comunidad científica y sus tesis e hipótesis propuestas de antemano.

En esta misma línea de crítica se ubican Tomas Kuhn, quien publicó en 1962 La estructura de las revoluciones científicas, con la cual "haría explotar" la racionalidad crítica popperiana. Según ésta teoría, el avance de la ciencia no se debe a su carácter acumulativo ni a la posibilidad de falsación de una hipótesis, sino a un largo proceso de crisis de la ciencia, que inicia con el descubrimiento de anomalías, la generación de crisis y, posteriormente, la búsqueda de normalidad de una nueva teoría o paradigma. En consideración a ello, la crisis se origina desde la ruptura, la discontinuidad, desde la posibilidad de ver el mundo de otra forma. Y esa mirada diferente solamente es otorgada por una comunidad de científicos que la van acordando.

En 1974 aparecen las críticas de Feyerabend contra cualquier método nomológico para organizar el conocimiento científico. El autor asume la idea de anarquismo metodológico, en la cual termina por privilegiar el oportunismo en la utilización de los métodos, pues, considera que los científicos han tenido que acudir a innumerables artefactos y estrategias, a veces secuentes, 
otras carentes de cualquier secuencia lógica, para lograr el reconocimiento de sus teorías.

Lo importante en este tangencial recorrido por el positivismo es que, desde las críticas al excesivo racionalismo positivista, va ganando espacio la intencionalidad del ser humano en el conocimiento científico y se va posesionando un imaginario que asume las pretensiones y construcciones de las personas como fundamental para el desarrollo de cualquier avance científico.

En el siglo XVII, Dilthey comienza a dar vida a una lógica que desafía el positivismo de Comte y el racionalismo de Descartes. Está fundamentada en el lenguaje, la historia y la tradición como escenarios de construcción de la realidad social, que sería desarrollada por la teoría del lenguaje, la fenomenología y la teoría crítica a la sociedad. Basadas en estas corrientes, e incluso en corrientes neopositivistas, en el siglo XX es fuerte el desarrollo de teorías que muestran las realidades sociales como de gran complejidad e imposibles de ser analizadas o estudiadas desde una consideración unidimensional.

Pese a las críticas fuertes a que ha sido sometido el positivismo y a la concepción actual casi generalizada de la complejidad de las realidades, incluso abióticaspreantrópicas, en la educación física, la mayoría de los fundamentos epistemológicos que sustentan sus estudios continúan amparados en imaginarios positivistas, en los cuales se escinde el cuerpo como soma, la motricidad como agregado, el sentimiento como subjetivo y la cultura como producto.

Es muy común leer estudios que buscan medir influencias funcionales del ejercicio, aislándolas de las dimensiones intencionales, o estudios que miden las características morfológicas sin considerar los escenarios culturales y sociales en que los individuos se encuentran.

\section{La complejidad de las}

\section{realidades de la educación física}

Varias tendencias relevantes surgen hoy en la consideración compleja de las realidades sociales y humanas: la teoría de la complejidad de Morin; la teoría cibernética de la sociedad, impulsada por autores como Frigogine - Capra; la teoría de los sistemas complejos, impulsada por Luhmann; la teoría de los sistemas vivos, de Maturana; las teorías de la acción comunicativa, de Habermas; o las teorías sobre imaginarios sociales, desarrollada por Durand, Castoriadis, Shotter o Pintos, entre otras.

La complejidad de la educación física se presenta en tha doble dirección: la naturaleza compleja de la educación y de la motricidad. De hecho, cuando nos referimos al campo de la educación física no nos enfrentamos únicamente a lo predefinido por la herencia biológica, sino $y$, sobre todo, a un campo cruzado por las pretensiones individuales de lo psicológico, las intencionalidades de lo social y la herencia cultural.

Desde el escenario de lo educativo, la educación física es construcción simbólica, devenida de las significaciones sociales que sobre ella se construyen. Esto es, que lo que se enseña y aprende en la educación física está condicionado a los acuerdos sociales construidos sobre lo que debe y no debe ser la clase. Lo anterior implica un reto importante para la investigación, puesto que se debe indagar justamente sobre las construcciones sociales que definen que una clase de educación física sea o no de calidad. Y la calidad es construcción y acuerdo social devenido de los procesos comunicacionales que se viven a diario. La calidad no es igual para todo el mundo, ya que para algunos estará relacionada con el logro y perfección de algunos gestos técnicos, mientras que para otros la buena clase será la que permita grandes procesos de reconocimiento social, así no se logre el desarrollo exagerado de destrezas deportivas.

Es claro que el objeto de la educación física es la motricidad como expresión paidológica. Pero no referida al estudio de la motricidad, sino a la educación del ser humano en la motricidad y desde la motricidad. En realidad, la motricidad no es expresión independiente de todo el ser humano, sino que forma parte de la humanidad como síntesis del movimiento intencional y recoge la naturaleza de todo lo que el ser humano social y particular es y llega a ser.

Sin embargo, la relación que se funda en una lógica trascendente devenida del cartesianismo es la relación cuerpo-motor; y no cuerpo-motricidad. Esta lógica se construye desde el cuerpo como lo somático, lo que está constituido para ser habitado; porque quien habita es quien da vida. De hecho, esta perspectiva es también herencia de los imaginarios judeocristianos, que permitían ver el cuerpo como el medio, el cascarón habitado, utilizado para que el alma expresara la presencia divina.

En esta perspectiva, lo motor es instrumental, lo que produce movimiento, es la "máquina que traduce la energía en movimiento"; ni siguiera es la energía, sino la máquina, que lleva a que esta energía se concrete en actividad. Un motor es un vehículo de la energía. Esta categoría encuentra relación con la idea de que lo motor en el cuerpo humano es esa máquina que lo hace funcionar; una máquina compuesta por partes fundamentalmente biológico-funcionales y, por tanto, controlables, medibles y reductibles a la lógica causa-efecto. 
Asumir lo motor como categoría central de la educación física es aceptar la dualidad del ser humano, prevista por el positivismo, pero criticada desde antes del siglo XVII. El avance en las discusiones sobre la naturaleza compleja de lo social y humano es un hecho en la actualidad, a tal punto que se presentan como simplistas las propuestas unidireccionales y nomológicas.

La educación física exige análisis y tratamientos más complejos. Por ejemplo, La corporeidad, esa estructura somática que no puede ser sin la psique, la cultura, la sociedad y las herencias biológicas, porque forma parte de ellas. La corporeidad habla del todo yo, sujeto a todas estas condicionantes que me hacen posible. No nazco siendo un sujeto corpóreo, sino que llego a serlo; en cambio, si nazco con un cuerpo, la calidad de corporeidad es una condición influida por múltiples mediadores y no solamente por la herencia genética y biológica.

Castoriadis encuentra esa dinámica efervescente y polifónica de la corporeidad cuando no habla de sujeto individual, sino de psicosoma, como ese conjunto de relaciones posibles ya mencionadas (Castoriadis, 1998: 28). La educación física, en sus términos, es producto de ese sujeto influido e influyente, y no de un individuo alejado de las contexturas de lo social, lo cultural, lo racional, lo imaginario.

¿No será que el concepto de cuerpo está saturado, puesto que ya no nos da a entender lo que queremos entender cuando se habla de este? ¿Acaso no será hora de tomar en serio las propuestas de Maffesoli (2004), de buscar otras categorías que no estén desgastadas y dejemos de buscar y acomodar categorías en escenarios en que ya no tienen sentido?

Merleau-Ponty (2000: 108), en su análisis de la fenomenología de la percepción, hace una digresión del concepto gastado de cuerpo. Considera, que no puede ser asumido, ni como un objeto, ni como una idea, pues el objeto lo es, en tanto permanencia, y esta no puede darse sin una ausencia. El cuerpo nunca puede ser ausencia, ya que su permanencia siempre está justo conmigo, siempre está en mí y para mí. Por eso, tampoco es idea ni puedo desplazarlo de mí, mirarlo fuera, ni puedo sustraerme de él para pensarlo; pero, con el hecho de ser de mí, existe como experiencia, puesto que el reflejo en el espejo no está libre de mis intenciones, ni el pensarlo, si fuese posible hacerlo desde otro cuerpo, estaría libre del cuerpo mismo.

El cuerpo es intencionalidad y experiencia, es ser del mundo, es identidad de lo humano y su expresión es la motricidad. Cuerpo como agregado, como funda, como prótesis de mí implicaría instrumentalización de mí mismo, reducción de la existencia humana a la función objetual, de permanencia y ausencia, implicaría desconocimiento de la existencia misma como seres humanos que nos transformamos permanentemente. Implicaría también reducción de la educación física a la funcionalidad de lo corporal.

En este mismo sentido de alteridad magmática, asumir el cuerpo como vida y mundo, como mi totalidad, es considerar la motricidad como expresión de corporeidad. Pues no es reflejo ni función de un cuerpo ausente o presente, no es un objetivo de la acción. Movimiento y corporeidad son origen y fin, son análisis y síntesis, sentimiento y experiencia; por eso, considerar la motricidad como lo meramente motor es una reducción de nuestra propia humanidad.

En este mundo globalizado, los cambios drásticos en presencia de lo corporal traen consigo cambios drásticos en la motricidad. No es el cuerpo material lo esencial en el mundo actual, sino la corporeidad dinamizada por la motricidad. Es el imaginario de cuerpo, es mucho más que la experiencia vital de lo presente como soma lo que prima en las relaciones de hoy. En consecuencia, la motricidad debe ser considerada expresión y sentido, ya que daría la connotación de corporeidad a la referencia de lo corporal como expresión de vida, como experiencia y mundo: las nuevas referencias y relaciones del cuerpo actual.

De ahí que cuando la referencia a la motricidad no sólo alude a la mecánica motriz, a la ejecución de movimientos, a su funcionalidad, sino también a la expresión de sentido de la corporeidad. No se hace referencia a la manifestación externa de lo corporal, a algo que existe como agregado a esta, sino a lo corporal mismo. De hecho, desde Merleau-Ponty (2000: 127), aunque se siga hablando de cuerpo, se asume una perspectiva de relación inmanente del movimiento con la vida; movimiento como totalidad, como dinámica expresiva de la corporeidad.

El fondo del movimiento no es una representación asociada o vinculada exteriormente con el movimiento mismo, es inmanente al movimiento, lo anima y lo lleva en cada momento; la iniciación cinética es para el sujeto una manera original de referirse a un objeto, lo mismo que la percepción.

En este sentido, el movimiento no puede ser tomado como representación, pues es la dinámica sentida, vivida, imaginada de la corporeidad que se expresa en sí y para sí en la motricidad.

Lo anterior significa que no podría existir la corporeidad sin la motricidad, ni motricidad sin corporeidad, 
es la corporeidad es enunciado y acción, y la motricid es expresión, enunciado y acción.

Un cuerpo humano sin movimiento es nada más que so: un cuerpo inerte. Los sistemas son similares a otros uerpos inertes, con algunas variaciones biológicas mí-mas; la corporeidad es mucho más que cuerpo, es exresión de la humanidad de cada individuo, es identihad y motricidad: la síntesis. La composición genética lo lemuestra, cuando define que el ADN del ser humano el de un Chimpancé son similares en $80 \%$. En el 20\% estante está lo que hace posible la corporeidad.

Por tanto, lo que vemos como expresión permanente e la corporeidad en la sociedad global es motricidad, bicada en un "horizonte de sentido", en términos de fadamer, o sea, en un entorno de relaciones diversas y terentes, que se percibe en el fondo del bullicio de la ida cotidiana.

La motricidad humana debe ser síntesis del ser humano, en cuanto dialéctica de la teoría y la práctica, de - imaginación: lo simbólico y la realidad como consrucción imaginaria que se edifica en los caminos de la zalización social. Manuel Sergio (1999), le da el estatus e sintesis cuando plantea la ciencia de la motricidad - mana desde la crítica al cartesianismo que ha sometitodas sus prácticas.

\section{La complementariedad etnográfica}

\section{como alternativa epistemológica e nvestigativa}

Es innegable la crisis de los imaginarios excluyentes que e amparan en el positivismo como única opción para studiar las ciencias bióticas-antrópicas.

Por tanto, una epistemología de la educación físia debe ampararse en la búsqueda de esos trazos que peneran las nuevas temporalidades, en la búsqueda de mevas formas de ser sujeto $y$, con ellas, las nuevas foras de relacionarse, ya que todas involucran el ser en - totalidad $\mathrm{y}$, por siguiente tanto, como sujeto motor, smo sujeto corpóreo. Quizá debamos reconocer la geralogía misma de la motricidad y los cambios que en sas construcciones discursivas se han generado y que o permiten verla de otra forma que la instrumental uminante.

Además, de la recuperación de la génesis misma de s categorías que constituyen la educación física, la zcreación y el deporte, debemos enfrentar esa trama magmática de lo biológico, sicosomático, cultural e hisirico. Esta consideración nos lanza a la complejidad tit las realidades que debemos abordar en los estudios relacionados con el área; la cual no podría ser abordada desde métodos nomológicos, pues estos darían al traste con la consideración verdaderamente compleja de la naturaleza de la educación física.

Por eso, se propone la complementariedad como alternativa que, reconociendo esa naturaleza compleja de la educación física, se apoya en diversas perspectivas teóricas y metodológicas para estudiar las realidades del campo. La complementariedad etnográfica (Murcia y Jaramillo 2000,2001) es más que la superposición de teorías y métodos de investigación; es ante todo un enfoque que reconoce la intencionalidad comprensiva de la investigación, accediendo desde ésta a las explicaciones contexturadas.

El diseño se elige entonces en relación con el objeto de estudio y el problema construido, y se apoya en las teorías que puedan otorgar claridad respecto de la comprensión de este objeto y problema. En consecuencia, el enfoque de complementariedad no es un método de investigación, una superposición de teorías o métodos. Es ante todo comprensión y construcción del objeto y del problema, y desde estas, definición de un diseño que utiliza los métodos de recolección que considere necesarios para la comprensión profunda e incluso amplia del problema (Murcia et ál., 2005; Jaramillo, Murcia y Portela 2005; Murcia, Orrego y Portela, 2005; Murcia, 2006, 2007).

La investigación en educación física amerita un análisis profundo no solo de la naturaleza del objeto a investigar, sino también del interés del investigador, puesto que el interés debe tener correspondencia con el objeto y el problema. O sea, no es posible investigar un problema referido a lo que hace que los estudiantes no les agrade la clase de educación física, o no practiquen un deporte determinado, o que se retiren antes de tiempo de una prueba, o que no se comprometan profundamente con un equipo, asumiendo un interés técnico. Ya que ese problema no tiene que ver con las explicaciones causa-efecto, sino con las razones y los imaginarios sociales que se tienen y se construyen al respecto.

La investigación en educación física debe partir fundamentalmente de una razón epistemológica porque, si bien es importante la claridad de antecedentes y los apoyos teóricos respecto del problema que voy a solucionar, la comprensión del problema para construir el objeto de estudio no se puede lograr si no se aborda el problema desde las consideraciones de su naturaleza social y cultural, en el marco del interés del estudio y el enfoque de investigación. 
Por ejemplo, un estudio sobre las reacciones químicas de un componente sobre otro exige un control experimental con un claro interés técnico, pero no exige análisis epistémico del problema. Sin embargo, si el estudio involucra lo antrópico, o sea ese ser humano como cultura, el estudio requiere las explicaciones y justificaciones epistemológicas sobre la naturaleza del problema. Si el estudio busca analizar los efectos de un componente químico sobre el rendimiento deportivo, involucrará la corporeidad y la motricidad, y no sólo el cuerpo y el movimiento como soma y energía. Por tanto, el interés no puede ser meramente técnico; por ejemplo, medir las consecuencias fisiológicas, porque las reacciones fisiológicas son producto de un sujeto que piensa, siente, interactúa; de un sujeto motricio y, por tanto, fenomenológico e imaginativo. Las reacciones estarán asociadas a unos comportamientos sociales, culturales, sicológicos, biológicos y somáticos que, al fundirse a manera de magma, esbozan lo que se produce como reacción fisiológica. El estudio debería analizar estas reacciones, en relación con las otras dimensiones que constituyen la naturaleza del ser.

Si esto es posible en el deporte, con mayor razón debe serlo en la investigación en Educación física, pues su objeto es la motricidad desde su paidología. Cuando se analizan los efectos de un programa, de una metodología o de unos mediadores sobre el aprendizaje, se involucran dimensiones psicológicas, pero también sociales, biológicas-genéticas, que hacen posible una forma de aprendizaje determinado. Así que no sería conveniente asumir que por estar relacionado con la educación, que es una dimensión meramente antrópica, deben olvidarse las dimensiones generales que cruzan el ser humano como totalidad compleja.

\section{Referencias}

Castoriadis, C. (1998). Hecho y por hacer. Pensar la imaginación. Buenos Aires: Eudeba.

Comte, Augusto. Semblanza filosófica. Recuperado de

htp//www.todoelderecho.com Port 80; Monografías.com

Descartes, René. Discurso del método y meditaciones metafísicas. Traducción de Manuel García Morente. Editorial Espasa Calpe, Colección Austral. El texto está disponible en la Biblioteca Virtual Miguel de Cervantes.

Feyerabend, P. (1987). Adiós a la razón. Madrid: Tecnos.

Foucault, M. (1975). Vigilar y Castigar. Buenos Aires: Siglo XXI Editores.

Foucault, M. (2003). Las palabras y las cosas: Una arqueología de las ciencias humanas. Buenos Aires: Siglo XXI Editores.
En la definición de lo que se quiere entra en juego la intencionalidad del estudio, el interés del investigador: si el interés es meramente técnico, debe sustentarse por qué y mostrar los argumentos que se tienen para no buscar las otras relaciones, lo mismo si es práctico o emancipatorio. En sí mismo, este análisis es epistemológico del objeto y del problema. Pero podría hacerse otro tipo de análisis que se salga de la condición de análisis epistemológico y se dirija hacia la genealogía de los conceptos. En este caso, la base de búsqueda son las formaciones discursivas, que ayudan a ver la emergencia de los objetos, y no las construcciones teóricas demostradas respecto del por qué una categoría es o no es, funciona o no funciona (Foucault, 1975, 2003).

En el primer caso, el afán es mostrar las relaciones, más o menos lógicas, que sostienen el concepto en términos de los apoyos científicos, o por tanto, la fuente para este análisis son las teorías demostradas y los argumentos científicos (lo que se ha llamado conocimiento científico sobre el problema). El segundo caso, la intención es desentrañar, desenterrar la génesis primera del concepto. Si se busca descubrir el porqué de la hegemonía de lo instrumental en la educación física, se podría acudir a un estudio arqueológico. La fuente para este análisis no estaría en la ciencia, sino en el saber (Foucault, 2003).

Si hacemos un análisis desde el origen del conocimiento, todo lo que lo aborda, las tendencias que se han dado, las teorías que sobre este se han generado, los métodos desde los cuales ha sido abordado, hablaríamos de epistemología del conocimiento (Kuhn, 2000). Pero, si no se hace este discurrir, sino que se busca en las formaciones discursivas para sustentar su aparición y su estado, hablaríamos de genealogía del saber. (DD

Jaramillo, L. G., Murcia, N. y Portela, H. (2005). La clase de educación física en Caldas. Un problema de preparación o seducción. Armenia: Kinesis.

Kuhn, T. (2000). La estructura de las revoluciones cientificas. Quinta reimpresión. Bogotá: Fondo de Cultura Económica.

Lakatos, L. (1974). Historia de la ciencia y sus reconstrucciones racionales. Madrid: Tecnos.

Maffesoli. M. (2004). Yo es otro. Debates sobre el sujeto: perspectivas contemporáneas. Comp. Laverde, M., Daza, G. y Mónica, N. Bogotá: Universidad Central- DIUC-Siglo del Hombre Editores.

Murcia, N. y Jaramillo, L. G. (1998). La danza, factor de promoción ético moral en jóvenes marginados. Revista de Educación Física y Deportes de España, Apunts, 54, 68-73. Barcelona. 
Murcia, N. y Jaramillo L. G. (2.000). Investigación cualitativa. La complementariedad etnográfica. Armenia: Kinesis.

Murcia, N. y Jaramillo L. G. (2001). Seis experiencias en investigación desde el principio de complementariedad. Armenia: Kinesis.

Murcia, N. et ál. (2005). Los imaginarios del joven ante la clase de educación física. Un reclamo por su reconocimiento. Armenia: Kinesis.

Murcia, N., Portela, H. y Orrego, J. F. (2005). La clase de educación física. Voces que reclaman visibilidad social. Armenia: Kinesis.

Murcia, N. (2006). Universidad y vida cotidiana. Memorias. Congreso Internacional de Pedagogía. Rudecolombia. Paipa.
Murcia, N. (2007). Imaginarios sobre universidad. Primer Congreso Internacional en Educación y Pedagogía. Bogotá: Universidad Pedagógica Nacional-CIUP. Publicado en separata especial.

Sergio, M. (1999). Um corte epistemológico. Da educação física a motricidade humana epistemologia y sociedade. Lisboa: Instituto Piaget.

Ponty, M. M. (2000). Fenomenología de la percepción. Quinta edición. Traducción de Jem Cabarnes. Barcelona: Ediciones Península.

Vasco, C. E. (1994). Tres estilos de trabajo en las ciencias sociales. $3 .^{2}$ edición. Documentos ocasionales, 54. Bogotá: Cinep. 